\title{
Superconducting nanobridges under magnetic fields
}

\author{
J.G. Rodrigo, H. Suderow, and S. Vieira* \\ Laboratorio de Bajas Temperaturas, \\ Departamento de Física de la Materia Condensada \\ Instituto de Ciencia de Materiales Nicolás Cabrera, Facultad de Ciencias \\ Universidad Autónoma de Madrid, 28049 Madrid, Spain
}

(Dated: November 1, 2018)

\begin{abstract}
We report on the study of superconducting nanotips and nanobridges of lead with a Scanning Tunnelling Microscope in tunnel and point contact regimes. We deal with three different structures. A nanotip that remains superconducting under a field of 2 Tesla. For this case we present model calculations of the order parameter, which are in good agreement with the experiments. An asymmetric nanobridge of lead showing a two steps loss of the Andreev excess current due to different heating and dissipation phenomena in each side of the structure. A study of the effect of the thermal fluctuations on the Josephson coupling between the two sides of a superconducting nanobridge submitted to magnetic fields. The different experiments were made under magnetic fields up to twenty five times the volume critical field of lead, and in a temperature range between $0.6 \mathrm{~K}$ and $7.2 \mathrm{~K}$.
\end{abstract}

PACS numbers: $\quad$ 61.16.Ch, 62.20.Fe, 73.40.Cg

\footnotetext{
${ }^{*}$ Corresponding author: S. Vieira

e-mail: sebastian.vieira@uam.es

Fax: + 34913973961
} 


\section{INTRODUCTION}

Twenty years after the invention by G. Binnig and H. Rohrer of the Scanning Tunneling Microscope (STM) 1], the scientific community has found a large diversity of interesting applications to this instrument. In addition to its capability of obtaining topographical information about conducting surfaces, the STM can also be used to make local vacuum tunneling spectroscopy measurements. It is frequent to find the acronym STM/S which indicates an experimental set-up that is optimized to accomplish both, microscopic and spectroscopic functions [2].

One of the most remarkable applications is the manipulation of atoms. The capability to have a finger in the atomic realm has permitted to a selected group of skilled scientists to build atom by atom well defined structures on well characterized conducting surfaces [3]. A fascinating world of new physical phenomena and technological developments is born, as envisioned by the gifted mind of R.P. Feynman already in 1959 [4].

The unprecedented control on the approach between macroscopic size objects that the STM has introduced, has made possible experiments going from the jump to the one atom contact, towards the evolution of connective necks of very small sizes [5, 6, 7]. The observed conductance steps and its relationship to the quantization of the conductance has triggered a considerable number of experimental and theoretical works. In some superconducting elements, as $\mathrm{Pb}, \mathrm{Nb}$ or $\mathrm{Al}$, measured at liquid helium temperatures, it has been possible, using the highly non linear form of the subgap conductance due to Andreev reflection processes, to relate the electronic conduction through a one atom contact to the number of transmitting channels [8]. This number turns out to be characteristic of the electronic structure of the connecting atom, in agreement with previous experiments and calculations for several normal metals [9].

A breakthrough in the world of nano-engineering has been the construction of chains of atoms of gold and the characterization of its mechanical and electrical properties [10, 11]. The forces involved in the process of formation and the dissipation mechanisms have been investigated in detail in this unique one-dimensional system. Recently, atomic chains of gold atoms connecting superconducting electrodes were also created an studied[12]. In this case, the electrodes were superconducting lead, on top of which a thin film (of thickness 20nm) of gold was evaporated, where superconductivity was induced by the proximity effect. 
In this paper we are going to focus on a superconducting nanostructure that has been experimentally studied in our group for the first time, and which consists of a superconducting nanobridge connected to two normal bulky electrodes [13, 14, 15, 16, 17, 18]. As we will show later on, the work of Prof. J.T. Devreese, to which this volume is devoted, and his group in Antwerp have contributed significantly to the understanding of its physical behavior.

\section{SUPERCONDUCTING LEAD NANOBRIDGES}

\section{A. Fabrication and general behavior under magnetic fields}

Approaching carefully the tip and sample of an STM by measuring the tunneling current for an applied bias voltage, it is possible to observe a clear jump in the conductance when the mechanical contact between tip and sample is attained. Pushing further on the tip into the sample, a neck of variable cross-section is formed. The area of the minimal cross-section can be estimated if the electronic mean free path is larger than the typical dimensions of the contact. In this case, transport is ballistic and Sharvin's formula applies. The neck can subsequently be elongated using the displacement capabilities of the piezo element connected to the tip, creating a nanobridge (NB) between both electrodes.

We have shown in previous papers that it is possible to induce order in the bridge by means of a mechanical annealing procedure [7, 14]. A sequence of pushing and pulling processes of small amplitude are superposed to a large, continuous pulling that defines the length of the nanobridge.

During the formation of the NB bias voltage is kept constant, and the current follows a staircase pattern that can be measured neatly during the small amplitude pushing and pulling cycles. Often, very reproducible patterns are measured, indicating that the neck evolves through very similar atomic configurations. Simultaneous measurements of the conductance and the force have shown that this staircase behavior of the conductance is due to a sequence of plastic and elastic deformations occurring within the neck [19].

Simple models have been proposed to obtain good information about the shape of the NB 14, 20]. As it is known, lead is a very ductile material which permits to obtain easily nanobridges as long as several hundreds nanometers. The nanostructure resulting from 
the fabrication process, which can be followed until the rupture of the neck, consists of two similar opposed cone-like sides. The NB can separated into two parts, and we obtain two tips of nanoscopic dimensions with an apex of atomic size. Due to the reduced atomic mobility at very low temperatures the nanostructures do not change its form with time. Figure 1 shows schematically the above mentioned stuctures, as well as a typical conductance curve obtained in tunnelling regime at $1.5 \mathrm{~K}$ and zero magnetic field.

We are going to address now our attention to the study of these structures when an external magnetic field is applied. It is well established on thermodynamical grounds that to destroy superconductivity with a magnetic field, the provided magnetic energy must equal at least the condensation energy, which is given by $\frac{1}{2} \mu_{0} H_{c}^{2}$, with $H_{c}$ the thermodynamic critical field. Bulk lead is a type I superconductor whose critical field is (at 0K) of about 800G. The characteristic superconducting coherence and penetration lengths are $\xi_{0}=51 \mathrm{~nm}$ and $\lambda=32 \mathrm{~nm}$ (also at $0 \mathrm{~K}$ and $\mathrm{H}=0$ ). Typical lead NB's have lengths several times larger than $\xi_{0}$, but its lateral dimensions are well below $\lambda$. In that case, there is no significant Meissner screening, and therefore the magnetic field needed to destroy superconductivity has to be much higher than the bulk critical field, $H_{c 1}$, in order to provide the required magnetic energy. In this way, a superconducting nanobridge (SNB) connected in a unique and perfect way to normal electrodes of the same material is obtained, when the applied magnetic field exceeds $H_{c 1}$. The transport properties of these type of SNB have been studied in detail, changing the diameter of the minimal cross section, the temperature and the magnetic field.

Here we present recent results in SNB's created under high magnetic fields. The process of formation and elongation of the nanobridge is continuously controlled by a monitorization of the I-V curves at each elongation cycle. When superconductivity nucleates in the nanobridge, characteristic superconducting features appear in the I-V curves, determining the actual creation of a SNB. We have measured the tunnelling density of states, after completely breaking a SNB created under a magnetic field of $2 \mathrm{~T}$, and we compare the results with some theoretical models. We also discuss two interesting experiments, where we focus on the very different superconducting behaviors found in asymmetric NB's due to the heating induced by the circulating current, and we demonstrate how thermal fluctuations influence superconductivity at nanoscopic dimensions. 


\section{B. Superconducting lead nanotips (SNT) at 2T: experiments and model calcula-}

tions

As a result of the rupture of a nanobridge, two, in principle equal, nanotips were formed. The one corresponding to the STM tip is moved to another position on the surface of the sample. The tunneling characteristics of this N-S junction give us directly the superconducting density of states of the nanotip, convoluted with the voltage derivative of the Fermi function. In Fig.2(a) we represent the evolution (at 2 Tesla) of the conductance curves of such a junction as a function of the temperature. Superconducting features disappear near 4.5K, indicating that the tip becomes normal.

These results can be understood quantitatively in the framework of the Ginzburg Landau calculations of Misko, Fomin and Devreesse 21]. These authors obtained in detail the spatial changes of the square of the order parameter when a magnetic field higher than $\mathrm{H}_{c 1}$ is applied, using a realistic geometry. In this way, they were able to map the amount of superconducting phase along the SNB. Their calculations have made clear how strong gradients of the superfluid concentration can be established at magnetic fields much higher than the bulk critical field. In this way, these authors significantly improved our first approach to the problem in which the nanobridge was modelled by a wire of a few nanometers in diameter[15].

Following the characterization in the tunneling regime, the SNT was brought into contact, with the same applied magnetic field (2 Tesla), without changing its geometry. This contact has a resistance of $400 \Omega$, and assuming that the conduction is in the ballistic limit and using Sharvin's formula, its can be estimated to correspond to a diameter of $0.8 \mathrm{~nm}$. Transport in this ballistic contact reflects the contribution of Andreev reflection processes, as it can be seen in Fig.2b, where we plot the conductance curves obtained at several temperatures between 0.8 and $4 \mathrm{~K}$

We have analyzed these experiments following the model of [16]. The magnetic field enters as an effective, position dependent pair breaking rate. The equations are solved selfconsistently, allowing to obtain a complete description of the superconducting behaviour of the SNT in terms of energy and distance to the tip apex. An important result of this theoretical approach is that, in agreement with experiments, a gapless regime is attained in the apex region already at a modest magnetic field. The proximity effect from the neighboring 
normal regions plays a crucial role to produce this singular situation, as it induces a finite density of states at the Fermi level at the tip apex. The SNT is represented as a conelike structure (inset of Fig.4) and the fitting parameters used in the calculation were $L_{\text {cone }}=200$ $\mathrm{nm}, \xi=25 \mathrm{~nm}, \mathrm{H}=2 \mathrm{~T}$, opening angle $=15^{\circ}$. The results of the calculation are represented in Fig.3a. We want to remark that once the parameters are chosen in order to fit the curve at the lowest temperature, they are kept constant along the subsequent temperature variations. The qualitative agreement is excellent. It is noteworthy to remark that our experiment can be also modelled using a BCS density of states with a pair breaking parameter $\Gamma$, as introduced in Ref.[22]. Fitting the curve at the lowest temperature we get $\Gamma=0.5 \mathrm{meV}$. This value is kept fixed in the whole temperature range (as $H$ is also kept constant), so we can infer the temperature dependence of $\Delta$. In Fig.3b we plot the conductance vs bias curves calculated within this analysis, and in Fig. 4 we plot the temperature dependence of $\Delta$ from both models. Note that the result in Fig.4 is basically the same within both approaches. However, there are tiny but relevant differences in the calculation of the conductance. The model of Ref. 16] gives much better account of the V-shaped characteristics observed in the experiments at low temperatures.

Note that the temperature dependence of $\Delta$ can also be obtained using the curves in the contact regime shown in Fig.3b. However, heating effects, which we treat below in more detail make a precise interpretation difficult.

\section{Asymmetric superconducting bridges under magnetic fields}

As a result of the process of creation a of a SNB, we can find also situations in which each half of the SNB presents a different geometry. The asymmetry produces specific features in the I-V curves measured in contact regime under magnetic field. An ideal curve in this case, without heating effects, would present Josephson current at zero bias, the associated subharmonic gap structure (related to $\Delta_{1}$ and $\Delta_{2}$ ), and finally a constant excess current

equal to $\frac{8}{3 e R_{N}} \frac{\Delta_{1}+\Delta_{2}}{2}$. A real curve, corresponding to an actual asymmetric SNB, will present complex features due to the heating effects resulting of a different power dissipation rate in each half. Thus, the expected loss of the excess current will take place in a two step process. One part of the SNB will become normal before the other as voltage and current (and therefore, power) are increased along the acquisition of the I-V curve. It is, therefore, the 
presence of heating the only way to unambiguously determine the existence of an asymmetric SNB.

The actually measured I-V curve at a high external magnetic field is somewhat more complex because it is the result of a competition between the two consequences of having a SNB structure. Sharpening the SNB produces an enhancement of superconductivity, but it also leads to an increase of the heating effects on the I-V curve due to a worse heat dissipation [23]. In fig.5 we present curves corresponding to two different SNB geometries obtained in a field of 0.6 Tesla, with the same normal state resistance. These results illustrate the above mentioned situation. In fig. 5a, curve A presents the highest value of Josephson current, thus it corresponds to the largest value for $\Delta$ in both sides of the neck, and therefore to the neck with the sharpest geometry. This SNB presents the largest heating effects, as observed in the two-step loss of the excess current which disappears completely at $4.8 \mathrm{mV}$ (fig. 5b). Curve B, with a lower Josephson current, corresponds to a less sharp SNB, with a better heat dissipation rate. Correspondingly, the observed loss of the excess current occurs at a higher voltage $(7.3 \mathrm{mV}$, see fig. $5 \mathrm{~b})$ than in curve A. The magnetic field behavior of these asymmetric bridges is shown in Fig.6. While the position of the feature corresponding to the first loss of the excess current (one of the sides of the SNB becoming normal) remains almost constant, we observe that the voltage at which the second loss takes place varies strongly with the applied field.

Note that this behavior is only observed under magnetic fields, where the different geometry of the two parts of the nanobridge gives two different spatial evolutions of the superconducting order parameter. Obviously the situation is more complicated, because the effective superconducting part of the nanostructure is also changing when the current increases during the heating process.

Clearly, it is possible to obtain relevant information about this nanostructure by a careful analysis of the observed characteristic curves. The capability of changing in-situ magnetic field, cross-section, temperature and bias voltage gives the possibility to study interesting non-equilibrium effects first reported in [17]. 


\section{Josephson coupling and thermal fluctuations in superconducting nanobridges}

under magnetic fields

We discuss now another aspect of our measurements, related to the Josephson effect in these nanobriges at $H>H_{c 1}$. A zero field study of these structures was previously reported

in ref.[24]. In superconducting bridges of resistances below a thousand $\Omega$, the Josephson coupling energy is much higher than the thermal energy in the relevant temperature range, and the Josephson feature at zero bias is very clearly measured. When the temperature is increased up to $T_{c}$ thermal fluctuations appear in a very narrow temperature interval close to $\mathrm{T}_{c}$. This leads to the appearance of a finite conductance at zero bias when we approache $\mathrm{T}_{c}$. In Fig.7 we show the zero bias conductance as a function of the reduced temperature for different magnetic fields. At each magnetic field, we have identified the corresponding $\mathrm{T}_{c}$ as the temperature where we loose superconducting features in the I-V curves. At low magnetic fields, the conductance rapidly increases when we cool below $\mathrm{T}_{c}$. But at higher magnetic fields (see Fig.7), when superconductivity becomes confined to a very small region around the neck, we can observe a finite zero bias conductance in a very large temperature range. This indicates that the magnetic field dramatically increases thermal fluctuations within the bridge. The change of size of the superconducting region, which has shrunk from the bulk value to a nanometric size superconducting bubble located at the center of the nanobridge, must be on the origin of this effect. More detailed experiments will be done changing the minimal cross section, the magnetic field, temperature and smallest cross section in well characterized bridges.

\section{SUMMARY AND CONCLUSIONS}

In this paper, we have shown a characterization of several lead nanostructures fabricated with the STM under magnetic fields. Experimental results and model calculations of the density of states of superconducting nanotips in the tunnel and point contact regimes have been presented. We have also discussed a series of features associated to asymmetric superconducting nanobridges under magnetic field. New and interesting effects associated to a non uniform heat dissipation in these structures have been observed. The Josephson effect in nanometric size weak links under magnetic fields and the role of thermal fluctuations was 
studied by measuring one of these structures as a function of temperature. The relevance of thermal fluctuations increases under magnetic fields.

In conclusion, we remark the good agreement between experiments and model calculations in these nanostructures. We also stress the relevance of this type of nanostructures for the future development of a new technology using elements of nanoscopic dimensions.

It is a great pleasure for the authors to contribute to this volume in homage to Prof. J.T. Devreesse. The discussions with Prof. J.T. Devreesse and coworkers have revealed to us many important aspects of superconducting nanostructures, a field where his contribution is of great relevance.

\section{ACKNOWLEDGEMENTS}

We also acknowledge discussions and helpful support from E. Bascones, W. Belzig and F. Guinea. Financial support from the ESF programme VORTEX and from the MCyT (Spain; grant MAT-2001-1281-C02-0) is also acknowledged. The Laboratorio de Bajas Temperaturas is associated to the ICMM of the CSIC.

[1] G. Binnig, H. Rohrer, Ch. Gerber and E. Weibel, Appl. Phys. Lett. 40, 178 (1982)

[2] See e.g. C.J. Chen, "Introduction to Scanning Tunneling Microscopy", Oxford Series in Optical and Imaging Sciences, Oxford University Press (1993).

[3] M.F. Crommie, C.P. Lutz, D.M. Eigler. Science 262, 218-220 (1993).

[4] "There's Plenty of Room at the Bottom" (1959) in Richard P. Feynman, Jeffrey Robbins. The Pleasure of Finding Things Out: the best short works. Penguin. 1999

[5] J.K. Gimzewski and R. Moller, Phys. Rev. B 36, 1284 (1987).

[6] N. Agraït, J. G. Rodrigo and S. Vieira, Phys. Rev. B 47, 12345 (1993).

[7] N. Agraït, J. G. Rodrigo, C. Sirvent and S. Vieira, Phys. Rev. B 48, 8499 (1993).

[8] E. Scheer, N. Agraït, A. Cuevas, A. Levy-Yeyati, B. Ludolph, A. Martín-Rodero, G. Rubio, J. M. van Ruitenberg and C. Urbina, Nature 394, 154 (1998).

[9] C. Sirvent, J. G. Rodrigo, S. Vieira, L. Jurczyszyn, N. Mingo, and F. Flores, Phys. Rev. B 53, 16086-16090 (1996). 
[10] Yanson et al., Nature 395, 783 (1998).

[11] G. Rubio-Bollinger, S. R. Bahn, N. Agraït, K. W. Jacobsen, and S. Vieira Phys. Rev. Lett. $87,026101(2001)$

[12] G. Rubio-Bollinger et al., submitted.

[13] J.G. Rodrigo, N. Agraït and S. Vieira, Phys. Rev. B, 50, 374 (1994).

[14] C. Untiedt, G. Rubio, S. Vieira and N. Agraït, Phys. Rev. B 56, 2154 (1997).

[15] M. Poza, E. Bascones, J. G. Rodrigo, N. Agraï, S. Vieira and F. Guinea, Phys. Rev. B 58, $11173(1998)$.

[16] H. Suderow, E. Bascones, W. Belzig, F. Guinea, S. Vieira Europhysics Letters, 50, 749 (2000).

[17] H. Suderow, S. Vieira, Physics Letters A, 275, 299 (2000).

[18] H. Suderow, E. Bascones, A. Izquierdo, F. Guinea, S. Vieira, Phys Rev. B, 65, 100519 (R) (2002).

[19] N. Agraït et al. Phys. Rev. Lett., 74, 3995 (1995); G. Rubio et al. Phys. Rev. Lett. 76, 2032 (1996); N. Agraït et al. Thin Solid Films, 253, 199 (1994).

[20] Z. Gai et al., Phys. Rev. B, 58, 2185 (1998).

[21] V.R. Misko, V.M. Fomin and J.T. Devreese, Phys. Rev. B, 64, 14517 (2001).

[22] R. C. Dynes, V. Narayamurti and J. P. Garno, Phys. Rev.Lett. 41, 1509 (1978).

[23] M. Tinkham et al. J. of Applied Physics, 48 , 1311 (1977).

[24] J.G. Rodrigo, N. Agraït, C. Sirvent and S. Vieira, et al. Phys. Rev. B 50, 12788 (1994).

[25] See e.g. A. Barone and G. Paterno, "Physics and Applications of the Josephson Effect", Wiley, New York (1982) and K.K. Likharev, "Dynamics of Josephson junctions and circuits", Gordon and Breach, 1986. 


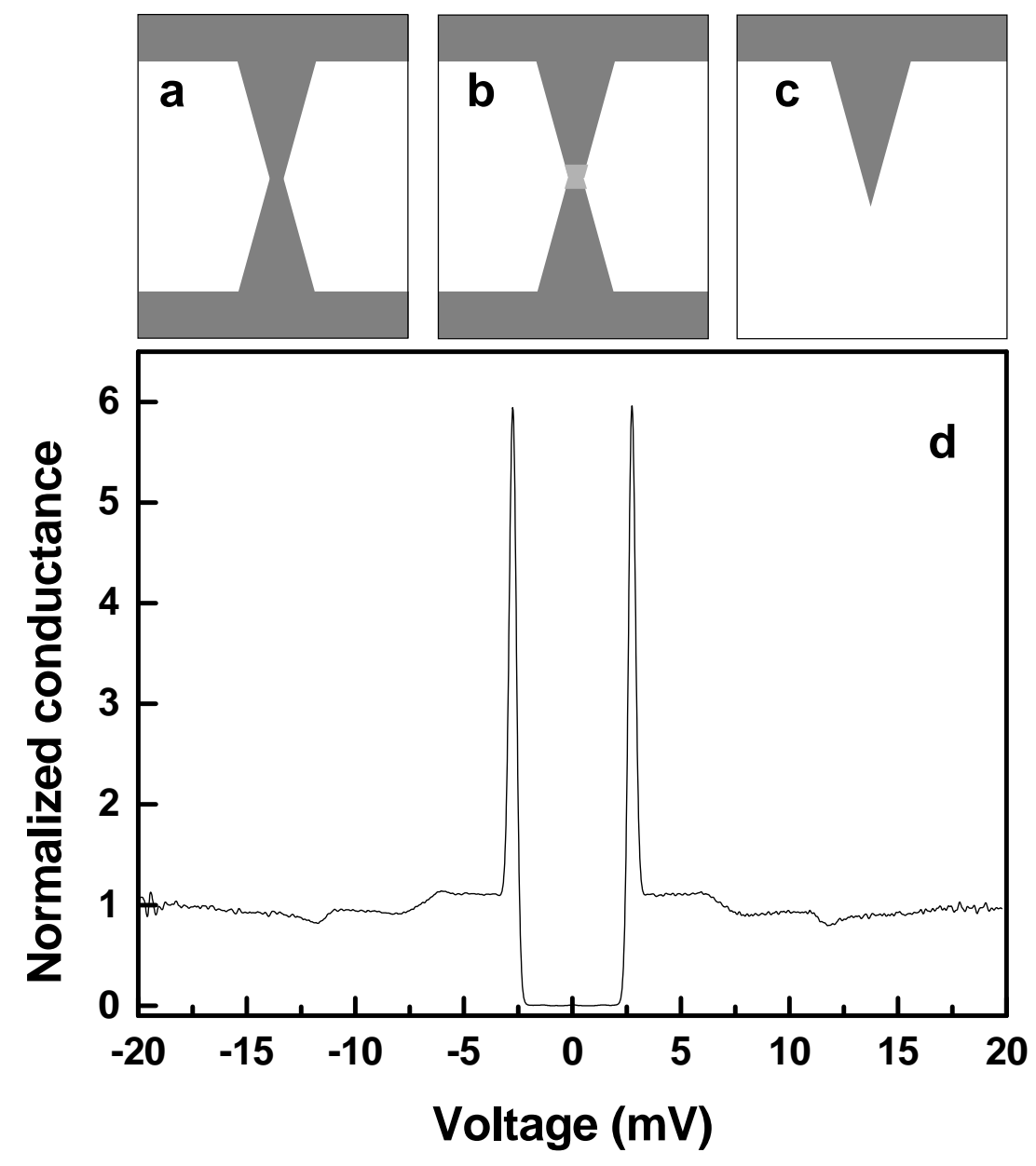

FIG. 1: The three main structures described in the text are shown schematically: a) nanobridge $(\mathrm{NB}), \mathrm{b})$ neck, and c) nanotip (NT). In d) we show a typical experimental conductance curve obtained in tunneling regime, in zero magnetic field, at $1.5 \mathrm{~K}$. (tunnel resistance is $\mathrm{R}_{N}=1.5 \mathrm{M} \Omega$ ). 


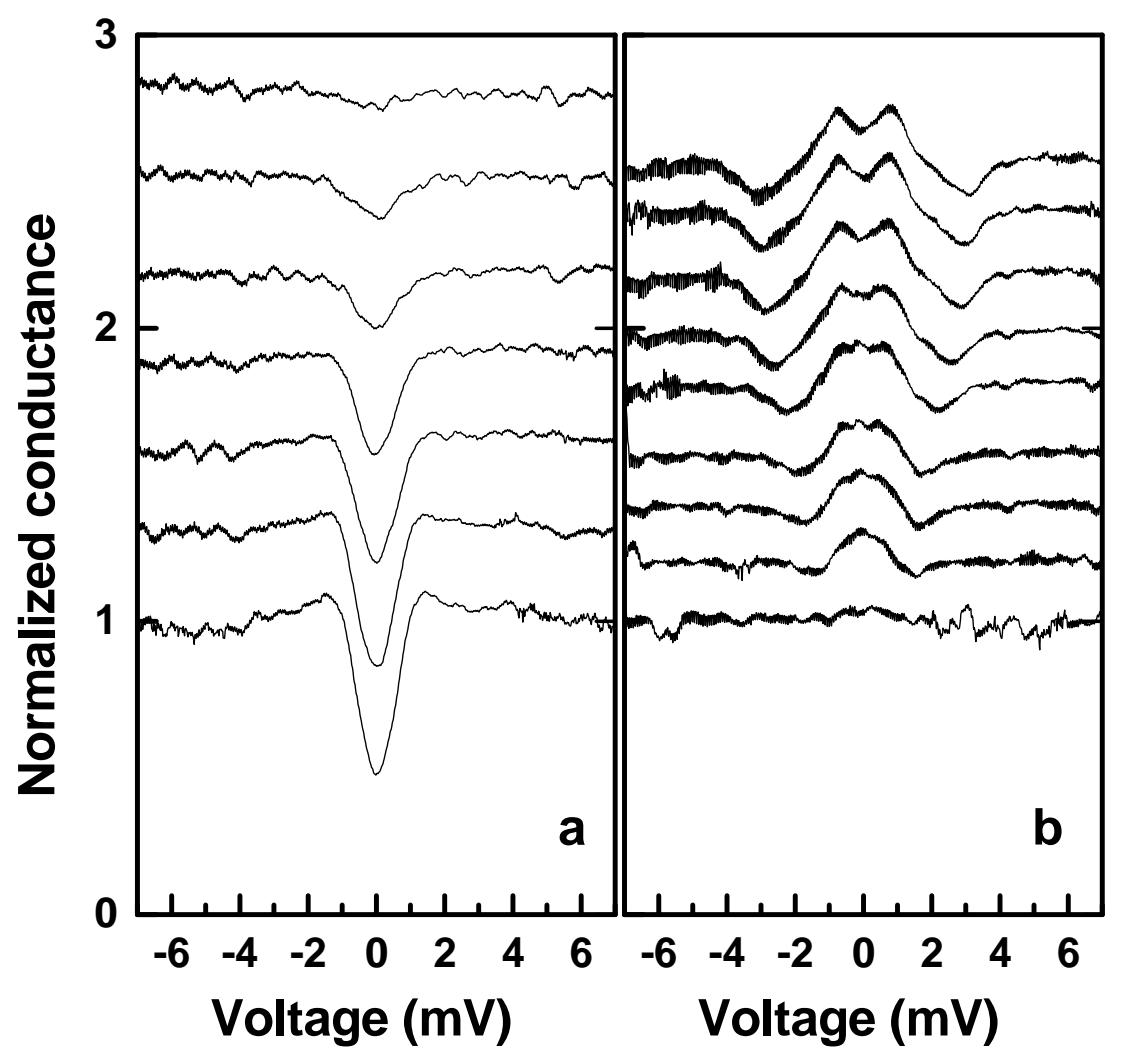

FIG. 2: (a.) Experimental conductance curves obtained in tunneling regime. The tip was sharpened and elongated as described in the text. The external magnetic field is 2 Tesla. Temperature is varied from $0.8 \mathrm{~K}$ to $4 \mathrm{~K}$, from bottom to top (curves are shifted for clarity). $\mathrm{R}_{N}=1.5 M \Omega$. (b.) Experimental conductance curves obtained in contact regime. Features due to Andreev reflections and heating effects are clearly observable. The external magnetic field is 2 Tesla. Temperature is varied from $0.8 \mathrm{~K}$ to $4 \mathrm{~K}$, from top to bottom (curves are shifted for clarity). $\mathrm{R}_{N}=400 \Omega$. 


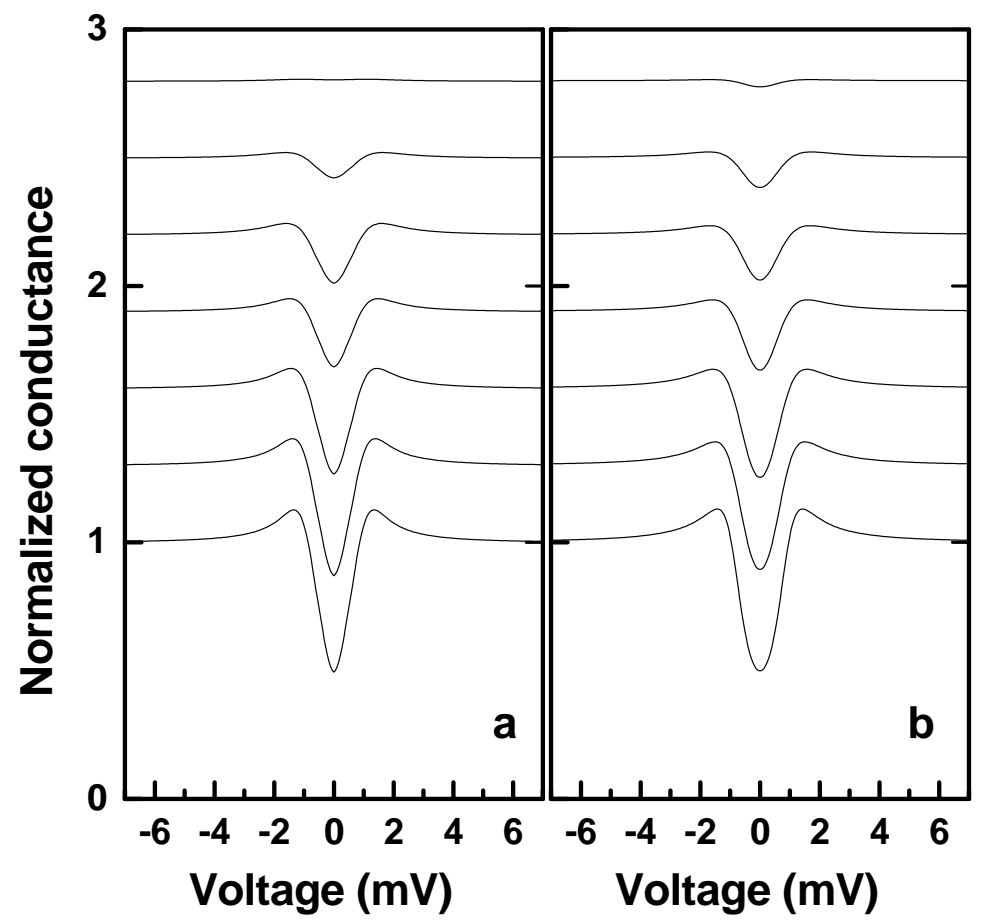

FIG. 3: (a) Conductance curves obtained using the formalism of ref. [16] and the parameters indicated in the text. After fitting the curve at the lowest temperature (bottom), all the parameters are kept constant except temperature, that is varied from $1 \mathrm{~K}$ to $4 \mathrm{~K}$ (in $0.5 \mathrm{~K}$ steps) in order to reproduce the experimental results (curves are shifted for clarity). (b) Conductance curves obtained after fitting the experimental curves in tunneling regime using the Dynes formalism. The pair braking parameter, $\Gamma=0.5 \mathrm{meV}$, resulting after fitting the curve at the lowest temperature (bottom), is kept constant for the rest of the curves. $\Delta$ is varied at each temperature (from $1 \mathrm{~K}$ to $4 \mathrm{~K}$, in $0.5 \mathrm{~K}$ steps) in order to fit the experiment. (curves are shifted for clarity). 


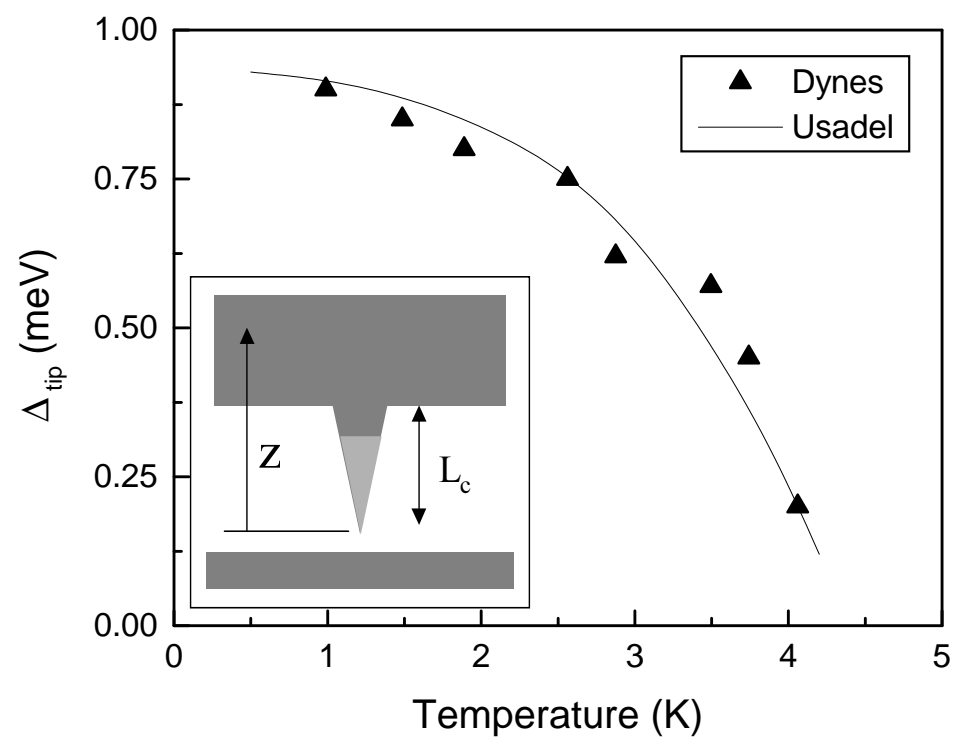

FIG. 4: Evolution of the superconducting gap at the tip apex vs temperature obtained from the different models described in the text. Inset: Model geometry used to reproduce the experimental evolution of the conductance curves vs temperature. The tip is modelled as a long and sharp cone attached to an infinite bulk electrode. The parameters used in the calculations were: $L_{\text {cone }}=200$ $\mathrm{nm}, \xi=25 \mathrm{~nm}, \mathrm{H}=2 \mathrm{~T}$, opening angle $=15^{\circ}$. 

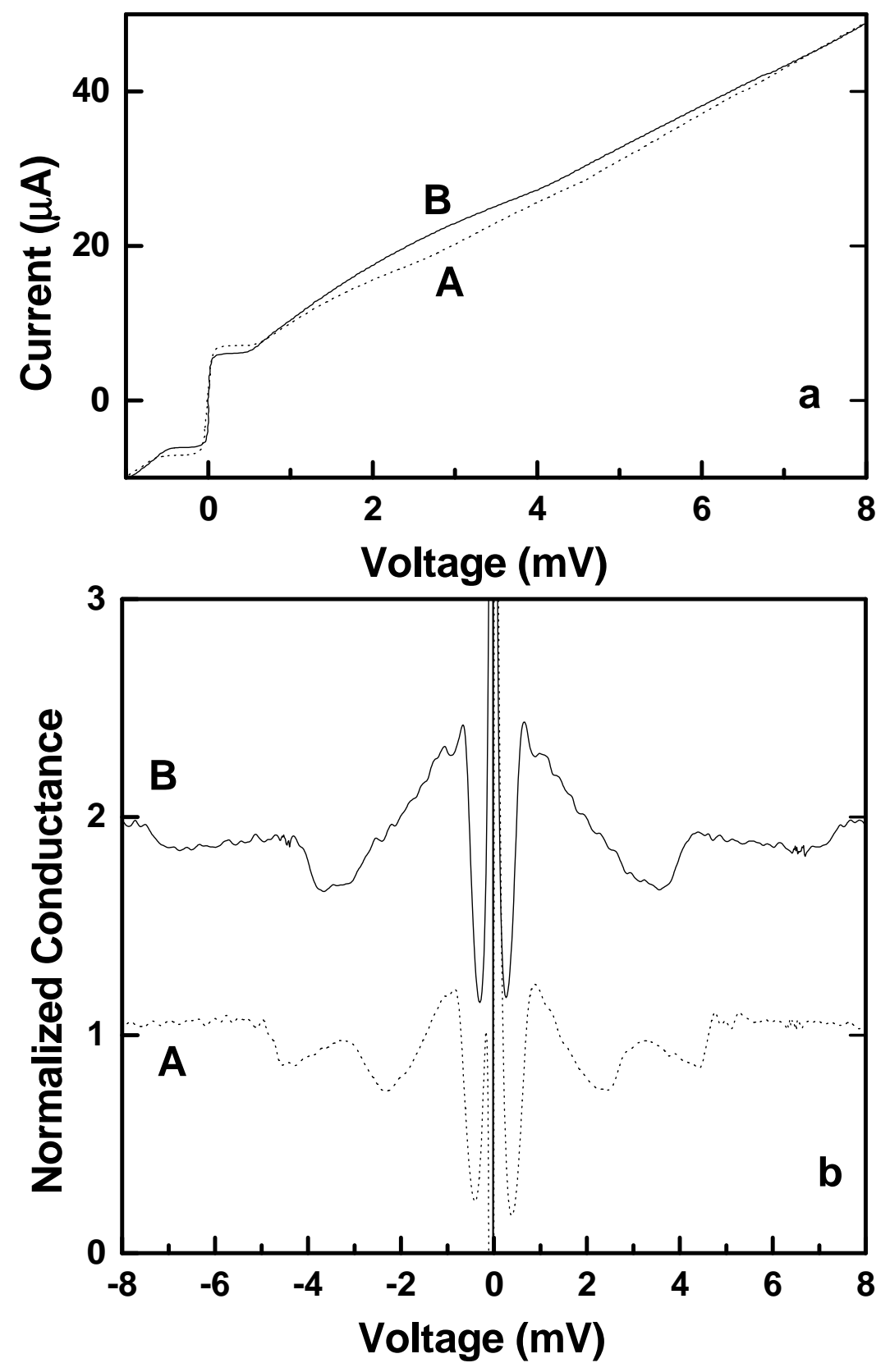

FIG. 5: I-V curves (a) and Conductance curves (b) obtained in contact regime, in the presence of a magnetic field, $\mathrm{H}=0.6$ Tesla, at $1 \mathrm{~K}$, corresponding to two different asymmetric nanobridges. The nanobridge with the sharpest geometry (A, dotted line) presents an enhanced superconductivity (larger Josephson current) and larger heating effects (worse dissipation).In both cases $\mathrm{R}_{N}=180$

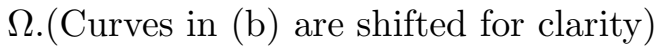




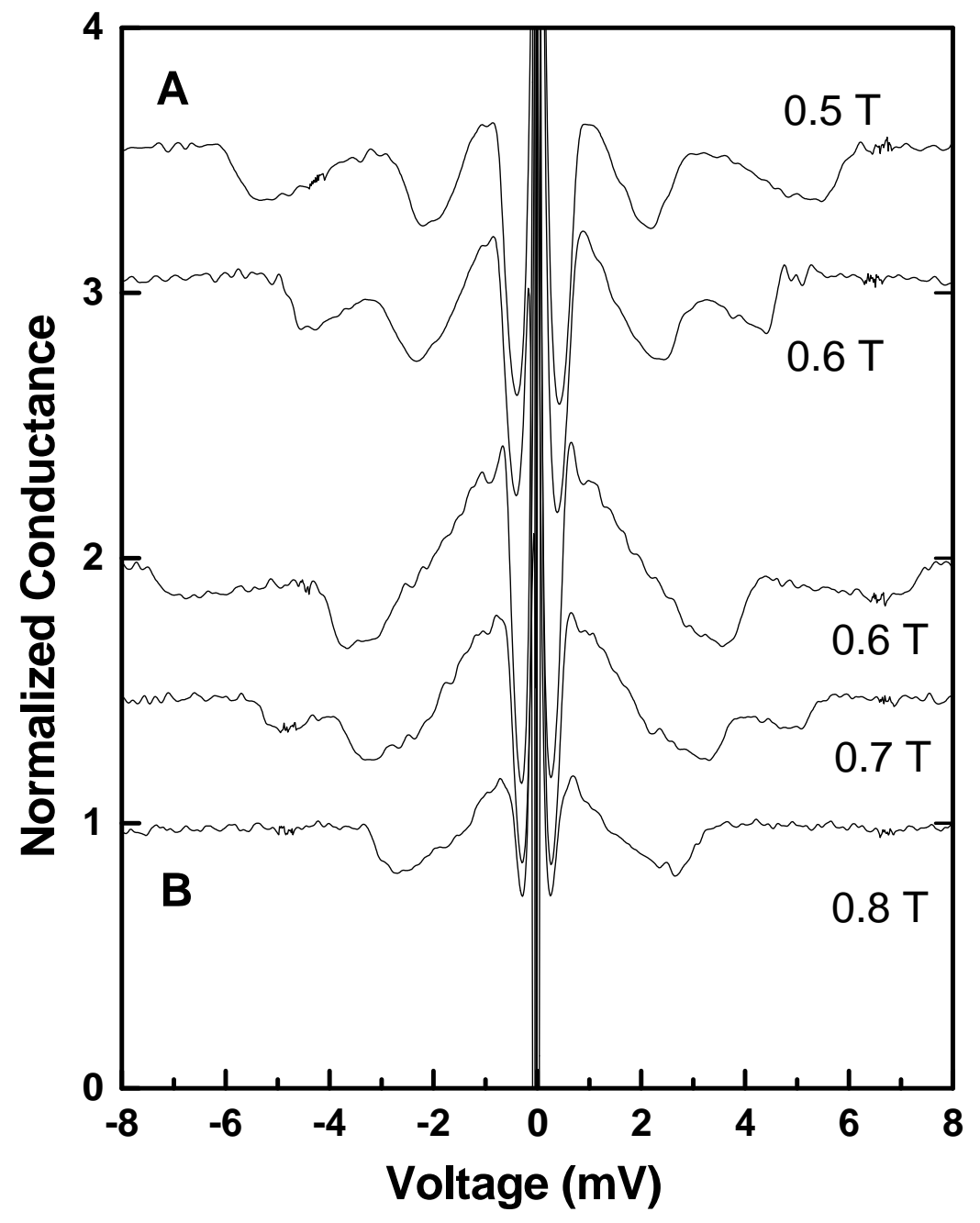

FIG. 6: Conductance curves obtained at different magnetic fields for the two nanobridges presented in fig.5. In all cases $\mathrm{R}_{N}=180 \Omega$ and $\mathrm{T}=1 \mathrm{~K}$.(Curves are shifted for clarity) 


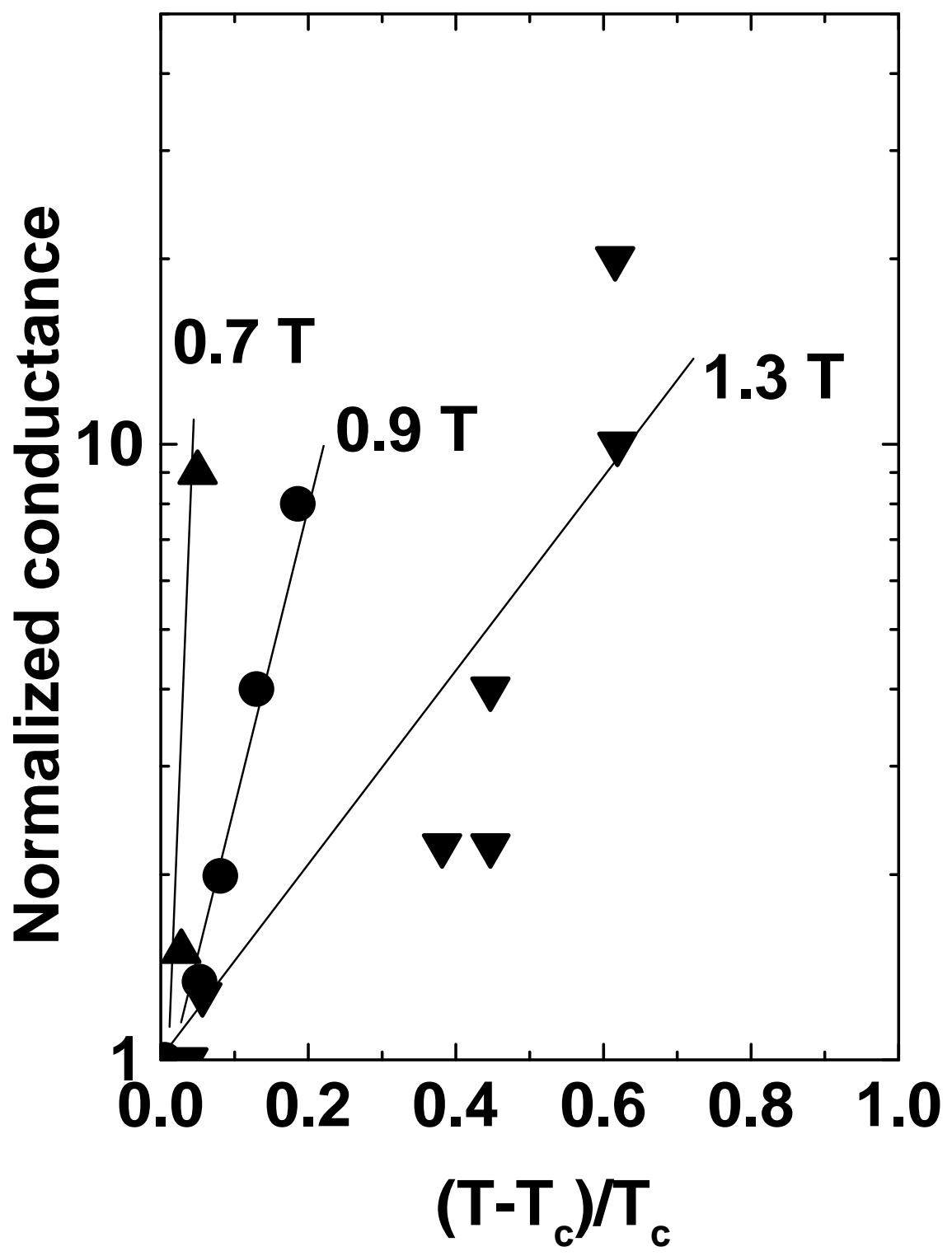

FIG. 7: Evolution of the zero bias conductance for a SNB as a function of the reduced temperature $\left(T-T_{c}\right) / T_{c}$, for various magnetic fields ( $T_{c}$ is measured for each field, see text; lines are guides to the eye). The smallest contact is of low resistance (about $100 \Omega$ ) and the SNB has a zero temperature critical field of about $1.7 \mathrm{~T}$. The temperature regime where phase fluctuations appear considerably increases at high magnetic fields. 